\title{
Reduction of drug-drug interaction and inappropriate prescription in polymedicated elderly who consult in the ambulatory
}

\begin{abstract}
Introduction: Older adults or elderly people over 64 years of age are patients more vulnerable to suffering adverse events related to medication, and this can generate states of both physical and psychological discomfort, loss of autonomy, mental disorders, etc. Objectives: To analyze the drug-drug interaction and inappropriate prescription of drugs in the outpatient setting in the elderly and implement barriers to reduce this problem.
\end{abstract}

Materials and methods: Quasi-experimental study, of the before after type. The Beers 2019 criteria were used to assess inappropriate drug prescribing. The Uptodate definition of drug-drug interactions and their classification were used.

Results: 203 polypathological, sarcopenic elderly patients were studied. These patients attended the outpatient consultation during the 2016-2018 period. 99 patients participated in the pre-intervention and 104 in the post-intervention. The distribution by sex was: 110 $(54.2 \%)$ women and $93(45.8 \%)$ men. The mean age was $77.3+13.3$ years. In the total sample analyzed before the intervention, $33(33.3 \%)$ showed inappropriate prescription of drugs criteria. Drug interactions in the pre-intervention were present in 51 patients $(51.5 \%)$. After the intervention that consisted of training doctors with the Beers criteria and editing a list with clinically relevant interactions in the elderly, which can cause adverse events, inappropriate prescription was reduced to 26 patients $(25 \%), p=0.05$, and clinically relevant interactions were reduced from $51(51.51 \%)$ to $12(11.53 \%), \mathrm{p}=0.003$. The association between inappropriate prescription and clinically relevant interactions is OR: 3.23 (95\% CI 1.91-3.88).

Conclusions: The proportion of patients with inappropriate prescription is within the ranges published by various authors as well as drug interactions, the intervention was good to reduce the two problems in this sample of patients.

Keywords: inappropriate prescription, elderly, drug-drug interaction
Volume 6 Issue 4 - 202I

\section{Fajreldines A}

Aleman Hospital,Austral University Hospital,Argentina, Buenos Aires, Argentina

Correspondence: Fajreldines A, Aleman Hospital, Austral University Hospital, Argentina, Buenos Aires, Argentina, Email fajreldinesa@gmail.com

Received: July 06, 2021 | Published: December 10, 2021

\begin{abstract}
Abbreviations: ADE, adverse drug events; PIM, potentially inappropriate medications

\section{Introduction}

Globally, the proportion of people $>60$ years of age is increasing faster than that of any other age group. Gender disparities, structural changes, changes in the burden of disease, drug therapy, risk of disability, changes in lifestyle, economic factors, among other elements, indicate the importance of evaluating global health care and the specific problems of this population. ${ }^{1}$
\end{abstract}

The organic changes that affect the elderly such as the reduction of drug metabolism, the amount of free drug in plasma, alterations in absorption among others, change the distribution, metabolism and excretion of drugs, constituting the elderly, in a population susceptible to adverse drug events (ADE). ${ }^{2}$

Medications whose risk of adverse drug events (ADE) in the elderly or elderly exceeds expectations of clinical benefits compared to more effective, safe and available alternatives are called potentially inappropriate medications (PIM). ${ }^{3}$

Polypharmacy, a very relevant and necessary fact to treat multiple pathologies in this population, although a useful and necessary tool, is a fact that can unnecessarily over-medicate the elderly, increasing their morbidity and mortality, therefore the rationale for the prescribing act is of fundamental importance. ${ }^{4}$

A review mentions that there is a variety of methodological designs in the studies that analyzed polypharmacy, and determined that this is a multifactorial process, and inappropriate prescribing is associated with negative health outcomes, such as a higher frequency and types of adverse events and of drug interventions involving different classes of drugs. ${ }^{5}$

\section{Aims}

Analyze inappropriate prescription and drug interactions and intervene with a strategy to reduce this problem.

\section{Material and methods}

Estudio quasi experimental de tipo antes y después. The patients included were adults over 64 years of age with polymedication and multiple pathologies who consulted in the outpatient setting of a medical clinic, at the study center. The study was developed in a highly complex academic hospital in Argentina. Pluripathology was considered to be the simultaneous presence of two or more pathologies where it is not possible to determine which is the most relevant due to the supremacy of one over the other. ${ }^{6}$ 
The Beers 2019 criteria for inappropriate prescription of drugs were used6. The UpToDate definition and classifications of drug interactions were used, where serious interactions should be avoided, these are $\mathrm{A}, \mathrm{B}$ and $\mathrm{C}, \mathrm{D}$ and $\mathrm{E}$ are not relevant and were not included in this study. Type A interactions: are established and supported in clinical trials, the "B" probable and supported and the "C" suspected and supported, these three categories were called "clinically relevant".

Polypharmacy was considered to be the simultaneous presence of more than 4 drugs according to the WHO definition. ${ }^{8}$

The burden of disease was assessed with the Charlson index9, which predicts mortality and burden of disease according to preceding pathologies. Variables such as sex, age, self-medication habits and adherence to treatments by questioning the patient, pathologies, visits to more than two different specialists in less than six months, level of instruction (university, secondary, primary), residence-alone, were collected. with family or in nursing homes. Presence of cognitive disorder, chronic pain defined as the presence of pain of more than 3 months treated with or without medication. The independence in activities of daily living with the ABVD scale, with A being totally independent and $\mathrm{G}$ totally dependent and one point for each category $(1-7) \cdot{ }^{10}$

The investigators who analyzed the inappropriate prescriptions were two observers trained in the criteria, both of whom reviewed all cases. Concordance between the two reviewers was assessed with Cohen's Kappa test, obtaining a value of: 0.76 (95\% CI 0.74-0.81).

The intervention consisted of training with the Beers criteria to physicians and their dissemination and a list of clinically relevant interactions in the elderly and recommendations for their nonprescription that were also distributed in all clinics.

The data were loaded into Excel databases and processed with the SPSS 21 package, IBM ${ }^{\circledR}$.

Statistical significance was considered at $\mathrm{p}<0.05,95 \% \mathrm{CI}$, the calculated sample was made for known universes, with a precision of $3 \%$ and an alpha error of $5 \%$. The impact of the calculated effect was $70 \%$, and a factor of $72.3 \%$ was obtained ( $95 \%$ CI $68.1-75.4$ ).

\section{Results}

203 multiple pathological outpatient elderly patients were studied. There were 99 patients before the intervention and 104 in the postintervention. The distribution by sex was: $110(54.2 \%)$ women and 93 $(45.8 \%)$ men. The mean age was $77.3+13.3 y e a r s$. The most frequent diseases were: cardiovascular-55(27.9\%), respiratory-43(21\%), neoplasms-21 (10.3\%), neurological-12 (5.9\%), digestive-9(4,4\%), others $(30.5 \%)$. In the total sample analyzed before the intervention, 33 patients $(33.3 \%)$ showed inappropriate prescription of drugs criteria. Drug interactions in the pre-intervention were present in 51 patients $(51.5 \%)$. After the intervention, inappropriate prescribing decreased from $33.3 \%$ to $22.11 \%, p=0.08$, and clinically relevant interactions decreased from $51.51 \%$ to $11.53 \%$, $\mathrm{p}=0.05$.

Patients with inappropriate prescription before the intervention presented: indication of unnecessary non-steroidal analgesics (NSAIDs) $-13(13.13 \%)$, oral anticoagulants $10 \quad(10.10 \%)$, psychotropic drugs $8(8.8 \%)$, other $2(2.2 \%)$ and post-intervention: oral anticoagulants-12 (11.54), benzodiazepines-9 (8.65\%) and NSAIDs: 2 (1.92\%). The patterns of inappropriateness did not change before and after.

In an age-adjusted logistic regression, it was found that inappropriate prescription is related to the female sex, to the presence of moderate or severe pain and moderate and severe anxiety / depression, to consultation with more than one specialist, to the home in geriatric Table 1.

Table I Variables associated with inappropriate prescription and clinically relevant interaction

\begin{tabular}{lcc}
\hline Variable & OR & \%, IC 95\% \\
\hline female sex, & 4,32 & $(3,49-4,65)$ \\
presence of moderate or severe pain & 3,24 & $(3$, I2-4, I7) \\
moderate and severe anxiety / depression, & 2,89 & $(2, I I-3,45)$ \\
home in geriatric & $4, I I$ & $(3,43-4,76)$
\end{tabular}

Odd: Odd Ratio. IC: intervalo de confianza

The association between inappropriate prescription and clinically relevant interactions was 3.23 (95\% CI 1.91-3.88). It is observed that drug-drug interactions appear more frequently in patients with means of drugs per patient greater than 7 than less than $7,(35 \mathrm{vs} 11, \mathrm{p}=.0005)$.

\section{Discussion}

Drug interactions and adverse events remain a major problem worldwide among older adults. The results of the studies included in the aforementioned review may contribute to the improvement of advanced geriatric nursing practices, to promote the safety of older patients with polypharmacy. However, more research is needed to clarify the local knowledge gaps on the subject. ${ }^{11}$

$75 \%$ of the prescription was: NSAIDs, anti-aggregators, anticoagulants, hypo-cholesterol, anxiolytics, antacids, vitamins, anti-arthritics. Age influenced the number of drugs $(p=0.013)$ and drug-drug interactions $(p=0.039)$, with a positive correlation in the aforementioned study. Another similar finding is that as the age of the patients increases, the drugs that are prescribed increase, in patients aged 69-75years from an average of 5.6 drugs to 7.8 drugs in the range of 76-84years. ${ }^{12}$

In patients older than 84 years, the prescribed drugs amount to an average of 10.2 per patient. Elderly patients are at special risk for polypharmacy, both due to the prevalence of multimorbidity in old age and the uncritical application of the guidelines by physicians. ${ }^{13}$

The more medications a person takes, the greater the risk of drug interactions and unwanted side effects. Multiple aids and tools are available to ensure practical and reasonable drug monitoring so that the risks of drug interactions and unwanted side effects can be detected early and avoided. Although there are barriers to deprescribing for patients and prescribers, an issue not addressed in this study, its weakness, there are resources and strategies available that facilitate deliberate but judicious de-prescribing and deserve a broader application. ${ }^{14}$ This strategy is not widespread in our country, but we should educate or train prescribers to do it. ${ }^{15}$

\section{Conclusion}

Inappropriate prescription is relevant according to Beers 2019, and drug-drug interactions are related to it. We must work on this problem to reduce it, as in this study.

\section{Acknowledgments}

None.

\section{Conflicts of interest}

The author states there are no conflicts of interest. 


\section{Funding}

None.

\section{References}

1. Salech F, M Jara J, R Michea, et al. Physiological changes associated with aging. Revista Médica Clínica Las Condes. 2012;23:19-29.

2. Vallvué C, Seguridad y, medicamentos. Adverse drug reactions: methods and problems of pharmacovigilance. Barcelona:JR Prous; 1987.

3. Corsonello A, Pedone C, Lattanzio F, et al. Potentially inappropriate medications and functional decline in elderly hospitalized patients. $J \mathrm{Am}$ Geriatric Society. 2009;57:1007-1014.

4. Psicofarmacología geriátrica. Aging and disease. XIX Refresher course for postgraduate Pharmacy. Pharmacy faculty. Universidad de Navarra: Junio; 2000.

5. Price SD, Holman CD, Sanfilippo FM, et al. Association between potentially inappropriate medications from the Beers criteria and the risk of unplanned hospitalization in elderly patients. Ann Pharmacother. 2014;48:6-16.

6. The American Geriatrics Society 2012 Beers Criteria Update Expert Panel. AGS updated Beers Criteria for potentially inappropriate medication use in older adults. J Am Geriatr Soc. 2019;60:616-631.

7. Bates DW, Spell N, Cullen DJ. The adverse drug prevention study group. The cost of adverse drug events in hospitalized patients. JAMA. 1997;277:307-311
8. Web site of WHO World Health Organization.

9. Charlson ME, Pompei P, Ales KL, et al. A new method of classifying prognostic comorbidity in longitudinal studies: development and validation. J Chronic Dis. 1987;40:373-83.

10. Katz S. Assessing self-maintenance: Activities of daily living, mobility and instrumental activities of daily living. J Am Geriatr Soc. 1983;31:721726.

11. Kratz T, Diefenbacher A. Review psychopharmacological treatment in older people: avoiding drug interactions and polypharmacy. Dtsch Arztebl Int. 2019;116:29-30.

12. Gnjidic D, Johnell K. Clinical implications from drug-drug and drugdisease interactions in older people. Review. Clin Exp Pharmacol Physiol. 2013;40(5):320-325.

13. Scott IA, Hilmer SN, Reeve E, et al. Reducing inappropriate polypharmacy: the process of deprescribing. JAMA Intern Med. 2015 ;175(5):827-834.

14. Hima Bindu Gujjarlamudi. Polytherapy and drug interactions in elderly. $J$ Midlife Health. 2016;7(3):105-107.

15. Di Martino E, Provenzani A, Polidori P, et al. Evidence-based application of explicit criteria to assess the appropriateness of geriatric prescriptions at admission and hospital stay. PLoS One. 2020; 15(8): e0238064. 